\title{
La République des Lettres et les médecins en France à la veille de la Révolution: le cas d'Esprit Calvet
}

Laurence Brockliss

\section{Summary}

In the broad Republic of Letters of the second half of the eighteenth century, physicians played an important but singular role. The majority of them were forced to earn their daily bread, so only belonged to the Republic in their leisure hours. Inhabiting a double universe - the everyday world of their profession and the more refined world of their intellectual hobbies - they had to negotiate continually between the two. This liminal position of the citizen-physician is recaptured in this article through the example of a physician of the Midi, Esprit Calvet of Avignon (1728-1810). Calvet left a huge correspondence, thanks to which this duality between the practising physician and the citizen of the Republic of Letters can be studied in detail. On the one hand, this is a correspondence between the physician and his patients, augmented by letters between the physician and other physicians on medical topics. On the other hand, it is a correspondence between the physician and other men of science on non-medical subjects (archaeology, botany, bibliophily, poetry, etc.).

Keywords: Republic of Letters; medical correspondence; consultation by letters; Esprit Calvet

\section{Résumé}

Dans la grande République des Lettres de la deuxième moitié du dixhuitième siècle, les médecins jouent un rôle important, mais particulier. La plupart d'entre eux, contraints de gagner leur pain, n'appartiennent à cette République que pendant leurs heures de loisir. Habitant un double univers,

Prof. Dr. Laurence Brockliss, Faculty of Modern History, University of Oxford, Broad Street, UK-Oxford, OX1 3BD (laurence.brockliss@magdalen.ox.ac.uk). 
le monde quotidien du métier et le monde plus rare des passe-temps intellectuels, ils doivent continuellement négocier entre l'un et l'autre. Cette position liminale du citoyen-médecin est retracée ici à travers le cas d'un médecin du Midi, Esprit Calvet d'Avignon (1728-1810). Il laisse une vaste correspondance, grâce à laquelle cette dualité entre médecin praticien et citoyen de la République des Lettres peut être étudiée dans le détail. Il s'agit en effet d'une correspondance entre le médecin et ses clients malades, doublée d'une correspondance entre le médecin et d'autres médecins sur des sujets médicaux, et enfin d'une correspondance entre le médecin et d'autres gens de lettres et de sciences sur des sujets non médicaux (archéologie, botanique, bibliophilie, poésie, etc.).

\section{Introduction}

La République des Lettres, Etat fictif, mais dynamique, dura du début du $\mathrm{XVI}^{\mathrm{e}}$ jusqu'au milieu du XIX ${ }^{\mathrm{e}}$ siècle: ses citoyens étaient les érudits et savants de l'Europe. Son caractère pluriconfessionel, non spécialiste et progressiste lui donnait son identité spécifique. Quoique l'époque moderne fût une ère de division religieuse, les citoyens de la République des Lettres prêchaient une philosophie de tolérance. Chaque citoyen, tout en poursuivant ses propres intérêts intellectuels, reconnaissait la nécessité de se tenir informé sur n'importe quelle activité savante, et se dévouait généralement à plusieurs champs de recherche. Et quels que fussent ces derniers, il croyait que ses travaux étaient utiles à l'humanité en éclairant son ignorance ou en l'aidant à mieux maîtriser la nature ${ }^{1}$. A la fin du $\mathrm{XVI}^{\mathrm{e}}$ siècle, cet Etat n'était peuplé que de quelques centaines de personnes et était dépourvu d'une organisation visible. Un siècle plus tard, il s'était agrandi, mais ne contenait toujours pas plus de mille deux cents habitants ${ }^{2}$. Par contraste, à la veille de la Révolution française, cette République était devenue un empire intellectuel, qui pouvait se vanter de compter près de trente mille citoyens; ses activités étaient de plus en plus proclamées et encouragées par de nouvelles institutions, les académies scientifiques, dont cent environ furent fondées au cours du XVIII ${ }^{\mathrm{e}}$ siècle $^{3}$.

1 La meilleure introduction à la République des Lettres est celle de Bots/Waquet 1977; la meilleure étude d'une période spécifique est de Goldgar 1995.

2 Ultee 1987.

3 McClellan 1985. Le nombre de citoyens est ma propre hypothèse. Elle est fondée sur le fait qu'il y avait 2500 académiciens en France en 1789, que dans la vallée du Rhône une moitié des citoyens (au moins) n'étaient pas académiciens, et que la population de la France était un sixième de la population européenne au XVIII ${ }^{\mathrm{e}}$ siècle. 
De plus, dans la deuxième moitié du XVIII ${ }^{\mathrm{e}}$ siècle, on ne mesure plus l'influence et l'étendue de cette République par le seul nombre de ces citoyens. Jusqu'à la fin du XVII ${ }^{\mathrm{e}}$ siècle, le véhicule principal de communication entre les membres était la correspondance ${ }^{4}$. Bien que ses citoyens les plus en vue eussent dès le début fait circuler leurs idées sous forme imprimée, la plupart d'entre eux ne publiaient rien, ou sinon tardivement, même pour les plus éminents d'entre eux. Après 1750, la correspondance demeure toujours le médium de communication privilégié et le plus immédiat, mais la publication acquiert une nouvelle importance. Les citoyens les plus ambitieux cherchent désormais, à travers leurs propres publications ou à travers les pages des mémoires des nouvelles académies, à présenter leurs recherches à un public élargi. Et ce public n'est pas formé des seuls savants. Grâce au nombre croissant des gens éduqués, l'abandon du latin comme langue scientifique et le soutien accordé, pratiquement pour la première fois, aux activités des citoyens par l'Etat et l'Eglise, un million de lecteurs des deux sexes consomment désormais les travaux de la République sans participation active $^{5}$. De fait, il existe alors des citoyens cherchant délibérément leurs lecteurs hors de la République traditionnelle. Ceux que leurs ennemis appellent les «philosophes» ne se distinguent pas seulement par leur désir de soumettre le christianisme lui-même au scrutin des républicains, mais aussi par la cour pressante faite à ce public passif en publiant leurs critiques dans un style élégant et facilement lisible ${ }^{6}$.

Ainsi, la République des Lettres à la veille de la Révolution française embrasse les gens éduqués de toutes espèces - princes et gouvernants, gens de profession, négociants -, voire, quelquefois, leurs femmes. Même le paysan illettré n'échappe pas totalement à son influence. Pour faire des collections et accroître la somme de la connaissance, le citoyen actif a besoin d'agents de terrain, de travailleurs à domicile qui puissent lui révéler l'exis-

4 Dès le début du XVII ${ }^{\mathrm{e}}$ siècle, les citoyens se groupaient dans des cercles de correspondance, où un savant particulièrement dévoué se donnait la tâche de maintenir des contacts parmi des érudits de la région ou de l'Europe entière. Le premier à adopter ce rôle fut le parlementaire provençal Nicolas-Claude Fabri de Peiresc (1580-1737): voir Miller 2000.

5 Aujourd'hui on associe cette croissance au nombre des lecteurs avec le développement de la sphère bourgeoise publique, un espace où s'organise une critique de plus en plus sévère de l'Ancien Régime: voir Habermas 1989; Van Horn Melton 2000; Blanning 2002. Blanning souligne qu'en Grande-Bretagne et en Prusse, ce nouvel espace d'opinion publique était toujours dominé par la couronne. Pour le rôle des femmes dans la sphère publique, voir Goodman 1994.

6 Selon Broman, les philosophes ne faisaient pas partie de la République des Lettres parce qu'ils faisaient la cour au public. Voir Broman 1998. J'admets qu'ils aient été plus subversifs par rapport au statu quo que d'autres républicains de cette époque, mais eux-mêmes insistaient sur le fait qu'ils ne constituaient pas une secte à part, associant cet avis aux ennemis de la science tout court: voir d'Alembert 1887,73. 
tence d'une trouvaille ou lui chercher un minéral précieux. Il arrive souvent qu'un tel intermédiaire soit un ami ou un autre républicain aux intérêts analogues, mais c'est d'ordinaire un homme de peuple qui effectue la découverte initiale. Quand un paysan rapporte une urne funéraire qu'il vient de déterrer à son seigneur au lieu de la détruire, alors la République des Lettres a clairement envahi sa vie ${ }^{7}$.

Dans cette grande République des Lettres de la deuxième moitié du XVIII ${ }^{\mathrm{e}}$ siècle, les médecins jouent un rôle important. Même si la plupart d'entre eux sont simplement des consommateurs de ses travaux, il semble qu'ils aient été surreprésentés parmi ses citoyens actifs. Daniel Roche, étudiant les membres des académies provinciales en France, a montré que ces dernières contenaient plus de médecins que d'ecclésiastiques ou gens de loi, et que seuls les nobles étaient plus en vue ${ }^{8}$. Les médecins contribuent évidemment au développement de leur propre discipline, et, comme le démontre le cas de Carl von Linné (1707-1778), ils impriment leur marque sur les sciences associées à la médicine, comme la botanique et, plus largement, l'histoire naturelle. Mais, comme d'autres citoyens, ils ne sont pas spécialistes, et l'on peut trouver des médecins qui sont aussi antiquaires et historiens, voire poètes ${ }^{9}$. On rappellera que le chevalier de Jaucourt (1704-1780), qui étudia la médecine à Leyde avec Hermann Boerhaave (1668-1738), écrivit une grande partie de l'Encyclopédie ${ }^{10}$. En revanche, la plupart des médecins tiennent une position assez distincte, presque liminale, dans la République de l'époque des Lumières. En général, un citoyen actif a reçu une éducation universitaire et tire son aisance d'un revenu privé, même s'il a obtenu un diplôme professionnel ou possède un bénéfice. En conséquence, un tel citoyen peut, quel que soit son statut, s'adonner aux intérêts intellectuels sans interruption. Le citoyen-médecin n'est pas si chanceux. Souvent fils d'un petit marchand ou d'un chirurgien ou apothicaire, il est rarement riche. Il est en principe contraint de gagner son pain, et n'appartient à la République que pendant ses heures de loisir. Autrement dit, habitant un double univers, le monde quotidien de son métier et le monde plus rare de ses passe-temps intellectuels, il doit continuellement négocier entre l'un et l'autre ${ }^{11}$.

7 Une remarque faite par James Livesey, du Trinity College de Dublin, dans une conférence récemment donnée à l'«Enlightenment Workshop» à Oxford. Livesey prépare un livre portant sur les débats au sujet de la réforme de l'agriculture dans le Midi avant et pendant la Révolution française.

8 Roche 1978, vol.1, chap. 4.

9 Par exemple, Albrecht von Haller (1708-1777) et Erasmus Darwin (1731-1802).

10 Bien entendu, d'autres médecins qui contribuaient à l'Encyclopédie écrivaient au sujet de leur métier: voir Kafker 1996.

11 Brockliss/Jones 1997, chap. 8: sur le revenu du médecin provincial. 
Le présent article vise à démontrer la vérité de cette position liminale du citoyen-médecin en évoquant brièvement la vie professionnelle et scientifique d'un médecin du Midi, Esprit Calvet d'Avignon (1728-1810) ${ }^{12}$. Calvet (fig. 1) est le fils d'un apothicaire, Claude Joseph Calvet (1674-1743). Après avoir étudié ses humanités et la philosophie dans les collèges jésuites à Lyon et Avignon, il se voue à l'étude de la médecine à sa faculté locale et obtient

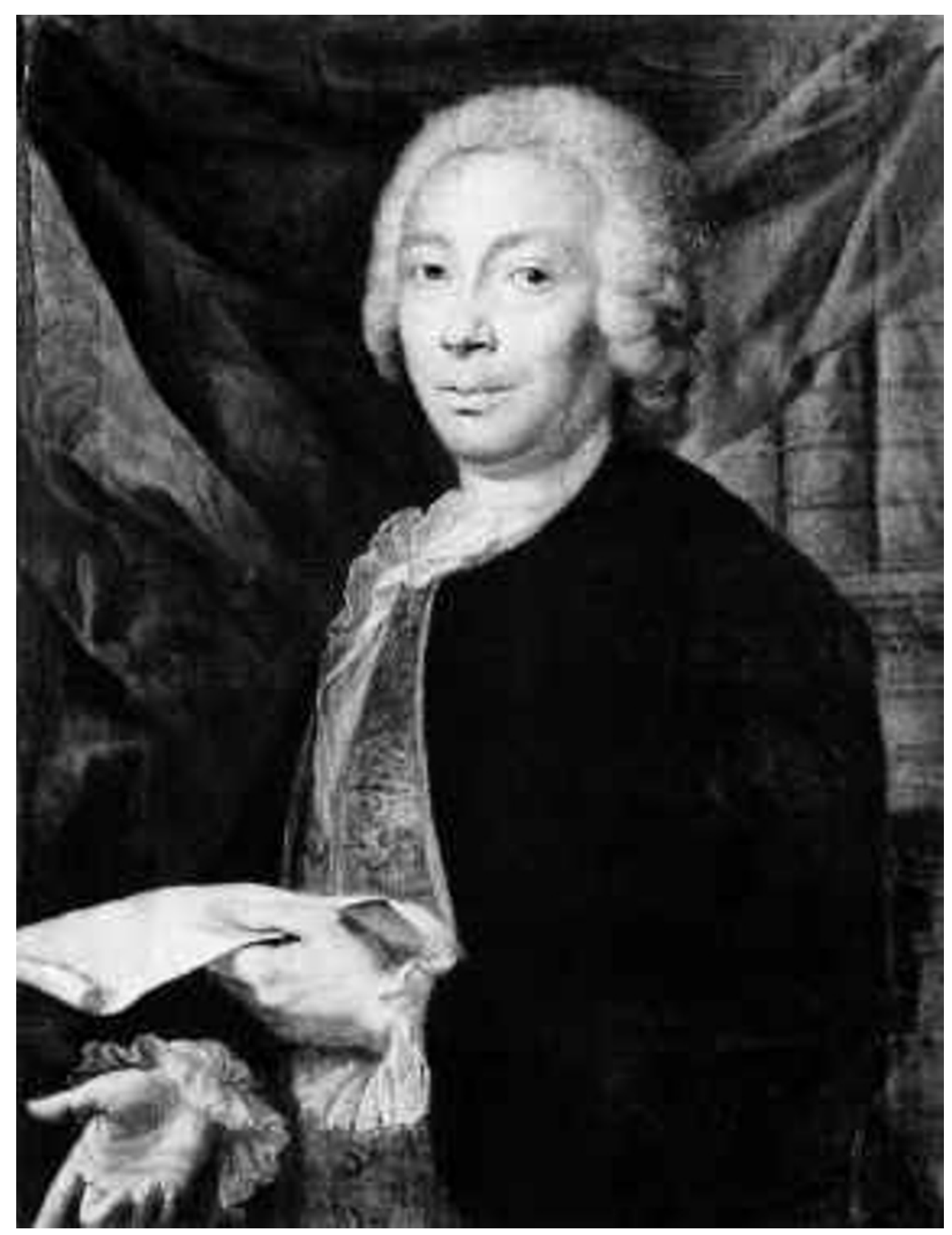

Fig. 1. Esprit Calvet, portrait à l'huile, attribué à Philippe Sauvan (1698-1792) (Musée Calvet, Avignon).

12 Pour les données biographiques sur Calvet, voir Brockliss 2002. 
son diplôme de doctorat en 1749. Il perfectionne ensuite pendant trois ans ses connaissances de la médecine et des langues anciennes à Montpellier et à Paris, avant de s'établir à Avignon comme médecin. Il y habitera jusqu'à sa mort, hormis plusieurs mois pendant la Révolution, et deviendra un médecin important dans la vallée du Rhône. Mais il est aussi bibliophile, antiquaire et naturaliste, qui appartient à plusieurs académies grâce à ses intérêts intellectuels plus larges et son empressement à faciliter les recherches des érudits déjà établis. Ainsi, après avoir aidé le comte de Caylus (1692-1765) à déterrer des objets intéressants dans le Midi pour le cinquième et le sixième volume de son Recueil des antiquités, on le fait membre correspondant de l'Académie des Inscriptions à Paris ${ }^{13}$. Calvet ne devint jamais un grand citoyen de la République des Lettres. Quoique bien connu en tant que collectionneur important et homme savant, il est trop timide pour publier ses idées et ne laisse qu'un seul imprimé ${ }^{14}$. De fait, on se souvient de lui aujourd'hui uniquement parce qu'il légua ses belles collections de livres, d'antiquités et d'histoire naturelle à la cité d'Avignon pour fonder un musée. Autrement dit, il est un républicain médiocre. Il demeure néanmoins un cas intéressant à étudier, parce qu'il laissa une vaste correspondance grâce à laquelle on peut explorer sa vie ${ }^{15}$.

Divisible en trois parties, cette correspondance révèle parfaitement les deux Calvet: le médecin et le républicain des lettres. C'est d'abord une correspondance entre le médecin et ses clients malades; c'est ensuite une correspondance entre le médecin et d'autres médecins sur des sujets médicaux; et c'est enfin une correspondance entre le médecin et d'autres gens de lettres et de sciences sur des sujets qui les intéressent mutuellement, mais ne concernent pas la médecine, sauf exceptionnellement quand un correspondant fait mention de sa santé et celle de sa famille. Cet article abordera chaque partie tour à tour.

13 L'Académie des Inscriptions, fondée par Colbert, était dédiée à l'étude de l'histoire ancienne. Tous les antiquaires éminents de la capitale en étaient membres. Anne-Claude-Philippe de Thubières était un esthète, qui espérait enseigner le bon goût aux Français par son Recueil d'antiquités égyptiennes, étrusques, grecques et romains (7 vols; Paris 1752-1767), où les illustrations des objets antiques trouvés en France servaient comme modèles pour des artistes et leurs patrons. Calvet était aussi membre correspondant des académies de Lyon, Grenoble et Volterra en Italie, et obtint en 1791 un fauteuil à l'académie de Marseille, bien qu'il ne fût pas un natif de la cité. Voir Brockliss 2002,31sq.

14 Calvet 1766.

15 Le Musée fondé par Calvet existe toujours, mais les papiers sont maintenant à la Bibliothèque Municipale d'Avignon (désormais BMA). Sa correspondance se trouve aux Mss 2350-2369, 3050sq., 4447, 5617 sq. et 5833. 


\section{La consultation par lettres}

Il est bien connu que les médecins des Lumières, surtout ceux d'une grande réputation, consultaient souvent par lettre. A une époque où le malade était libre de choisir n'importe quel médecin et où le médecin faisait son diagnostic après avoir écouté le malade raconter son histoire médicale et non après avoir effectué un examen physique, une telle consultation avait un sens. Le client ou son représentant envoyait une lettre à un médecin important en décrivant sa maladie, et le médecin répondrait en donnant son diagnostic et proposant un régime et des remèdes. Malheureusement, la consultation par lettre reste toujours peu étudiée dans le cas de la France, à propos de laquelle il existe plusieurs articles sur une telle pratique, mais pas encore de monographie savante ${ }^{16}$. On peut expliquer partiellement cet état de fait par le contenu des archives survivantes: nous ne possédons normalement que la réponse du médecin, laquelle, de plus, habituellement manque le nom du malade quand ces consultations sont imprimées, soit par le médecin soit par ses amis et étudiants en vue de faire connaître au public ses capacités médicales. C'est pourquoi les consultations du médecin parisien Etienne-François Geoffroy (1672-1731) dans les premières décennies du siècle sont si intéressantes; les cinq volumes qui se trouvent aujourd'hui à la Bibliothèque de la Faculté de Médecine de Paris contiennent les lettres originales aussi bien que les brouillons de sa réponse ${ }^{17}$.

Or, même si on ne possède que les lettres du médecin, on peut tirer grand profit de leur étude, comme les consultations de Calvet le démontrent. Calvet, médecin bien connu, avait fréquemment été consulté par lettre. Il ne subsiste que quelques lettres provenant des malades ayant recherché son avis, mais la Bibliothèque d'Avignon possède une belle collection - plus de 300 de ses propres consultations écrites - qui couvre les années 1765 à $1783^{18}$. Adressées aux malades, elles nous permettent de retracer l'étendue géographique de son renom, diverses données sur le niveau social de ses correspondants, ainsi que les contours de sa pratique médicale.

La carte 1 indique la provenance de ses malades. Il est évident le renom de Calvet se limitait presque exclusivement au niveau régional, puisque la plupart de ses malades habitaient à moins de 60 kilomètres d'Avignon, et

16 Pour une étude récente sur ce thème portant sur d'autre pays, voir Stolberg 2003. Voir aussi les références citées dans l'article de S. Pilloud, S. Hächler et V. Barras dans ce même volume, ainsi que Barras/Dinges 2005; Schnalke 1997; Duden 1987.

17 Bibliothèque de la Faculté de Médecine de Paris, Mss 5241-5245. Pour une introduction à cette collection, voir Brockliss 1994.

18 BMA Ms 5619, fos 738-859 (sans numérotation des feuilles entre ces deux feuilles). 


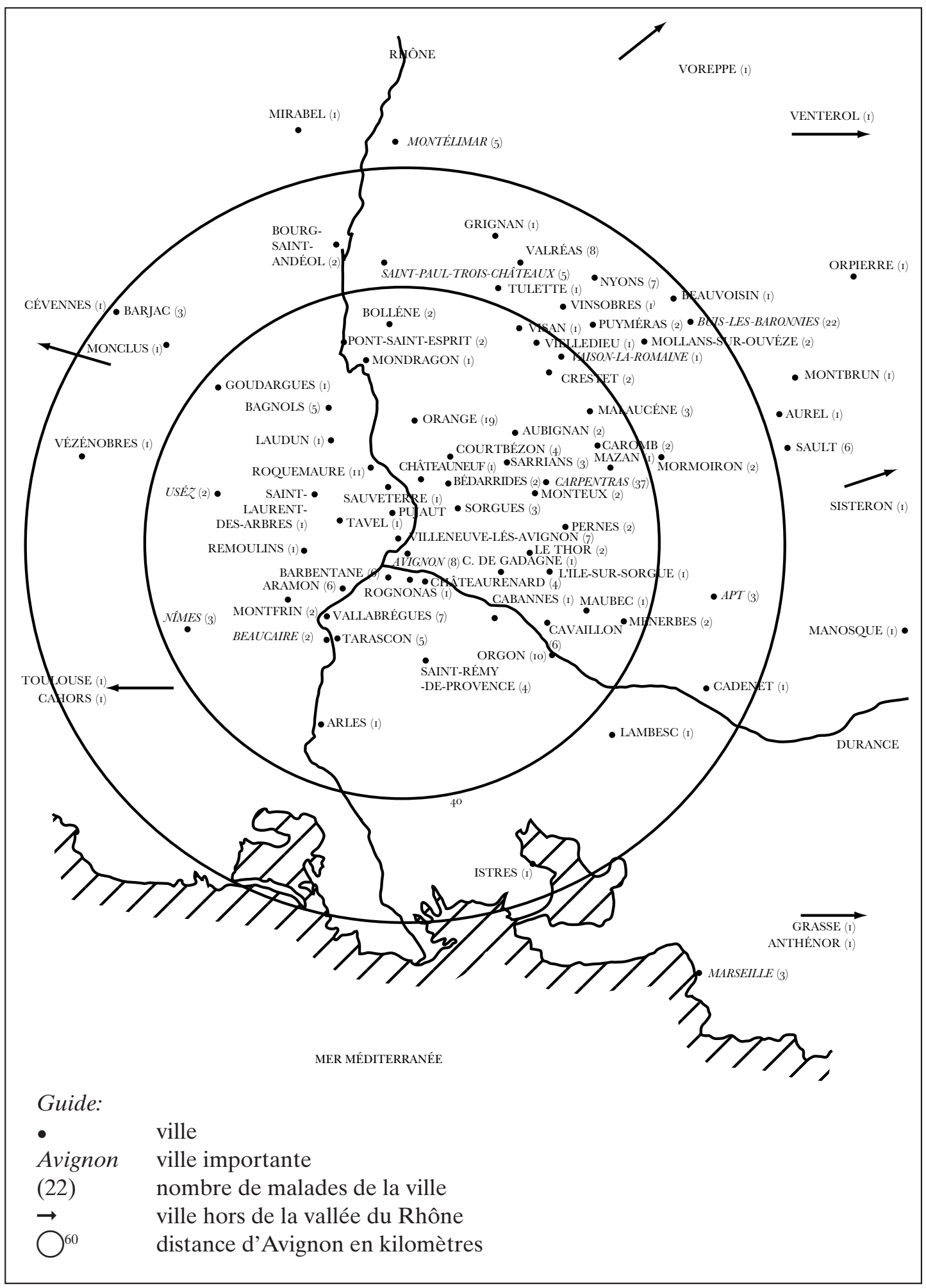

Carte 1. Provenance des malades de Calvet (Source: BMA Ms 5619, f ${ }^{\text {os }} 738-859$ : consultations écrites 1768 à 1783). 
parmi eux, en majorité à moins de 40 kilomètres à l'est du Rhône et au nord de la Durance. Calvet n'était donc même pas un médecin du Midi, sans parler de la France. De fait, lui-même avouait qu'il avait reçu une seule requête de consultation de Paris, dont il se montrait si fier qu'il inclut sa propre réponse à cette dernière dans son œuvre en six volumes manuscrits destinés à être publiés après son mort ${ }^{19}$.

La table 1 révèle le caractère social de ses malades. On voit que Calvet était consulté par les deux sexes, au nom des enfants, par les chrétiens, mais aussi par les juifs (alors nombreux entre Carpentras et Avignon). Parmi les

Table 1. Profil social des malades de Calvet (Source: BMA Ms 5619, fos 738-859: consultations écrites, 1768 à 1783).

\begin{tabular}{|c|c|c|}
\hline \multicolumn{2}{|l|}{ chrétiens } & 326 \\
\hline \multicolumn{2}{|l|}{ juifs } & 6 \\
\hline \multicolumn{2}{|l|}{ enfants } & 33 \\
\hline \multicolumn{2}{|l|}{ adultes } & 300 \\
\hline \multirow{2}{*}{ femmes } & & 136 \\
\hline & religieuses & 5 \\
\hline \multicolumn{2}{|l|}{ hommes } & 164 \\
\hline \multicolumn{2}{|r|}{ nobilité } & 6 \\
\hline \multicolumn{2}{|r|}{ bourgeoisie (rentiers?) } & 3 \\
\hline \multicolumn{2}{|r|}{ église } & 32 \\
\hline & évêque & 1 \\
\hline & chanoines & 6 \\
\hline & clergé inférieur & 18 \\
\hline & réguliers & 5 \\
\hline & maîtres & 2 \\
\hline & professionnel & 19 \\
\hline & officiers & 11 \\
\hline & avocats & 6 \\
\hline & médecins & 1 \\
\hline & maîtres (laïques) & 1 \\
\hline & commerce & 12 \\
\hline & artisans & 9 \\
\hline & domestiques & 3 \\
\hline & paysans & 2 \\
\hline & métier ou status inconnu & 78 \\
\hline totale & & 333 \\
\hline
\end{tabular}

19 BMA Ms 2347, fo 255 (sans date). Calvet fit deux copies de son œuvre: il en laissa une à Avignon, l'autre à Marseille; voir Brockliss 2002, 17. 
hommes, la plupart appartenaient aux professions libérales, en particulier à l'Eglise, mais il y avait aussi un petit nombre d'artisans, domestiques et fermiers. En mai 1771, Calvet écrit une consultation pour Plumay, un droguiste d'Orange, en avril 1773 pour Mlle Joure, une boulangère, et en janvier 1776 pour la femme d'un aubergiste de La Drôme. Ses clients étaient donc assez hétérogènes. A l'évidence, nombre d'entre eux ne contactèrent pas Calvet directement, mais sur la recommandation de leur médecin local. Les lettres sont souvent adressées au malade par un homme de l'art, en général un chirurgien. Autrement dit, la réputation régionale de Calvet était fondée en grande partie sur la confiance des médecins des petits bourgs et villages, qui faisaient sans doute valoir leur propre importance aux yeux de leurs clients en présentant leurs maladies au grand homme. De fait, la réponse de Calvet révèle que, souvent, les médecins lui écrivaient au nom d'un malade, non pas pour chercher son conseil mais pour obtenir de lui qu'il approuve les remèdes utilisés jusque-là ${ }^{20}$.

Le rôle du médecin local comme intermédiaire n'est guère étonnant. On le trouve dans la correspondance du parisien Geoffroy ${ }^{21}$. Plus inattendue est la découverte que beaucoup de clients consultent directement Calvet chez lui avant de recevoir sa lettre. Dans son cas, il semble que l'on ne puisse pas diviser clairement la consultation personnelle de la consultation par lettre. Les clients arrivaient chez lui munis d'une lettre d'introduction écrite par un médecin local décrivant leur maladie. Ils discutaient de leur problème avec le médecin avignonnais. Peut-être leur donnait-il un examen physique rapide. Puis ils s'en retournaient chez eux et attendaient sa lettre. On peut supposer que, pour Calvet, le seul moyen de maintenir un contrôle professionnel sur un client qu'il ne verrait plus jamais était de préparer une instruction écrite. Il paraît dès lors évident que la consultation par lettre a pu se continuer après l'avènement de la consultation physique au sens contemporain du terme après le tournant du dix-neuvième siècle.

\section{La correspondance entre médecins}

Pendant sa vie, Calvet reçut des lettres de 68 gens de l'art. Comme le montre la carte 2, la plupart habitaient la vallée du Rhône, mais provenaient d'une région beaucoup plus étendue que celle de ses malades correspondants.

20 Par exemple, le chirurgien Barjaval de Carpentras: voir Bibliothèque Municipale Carpentras Ms 1163, liasse séparée, sans date (c. 1780).

21 Brockliss 1994, 87-89. 


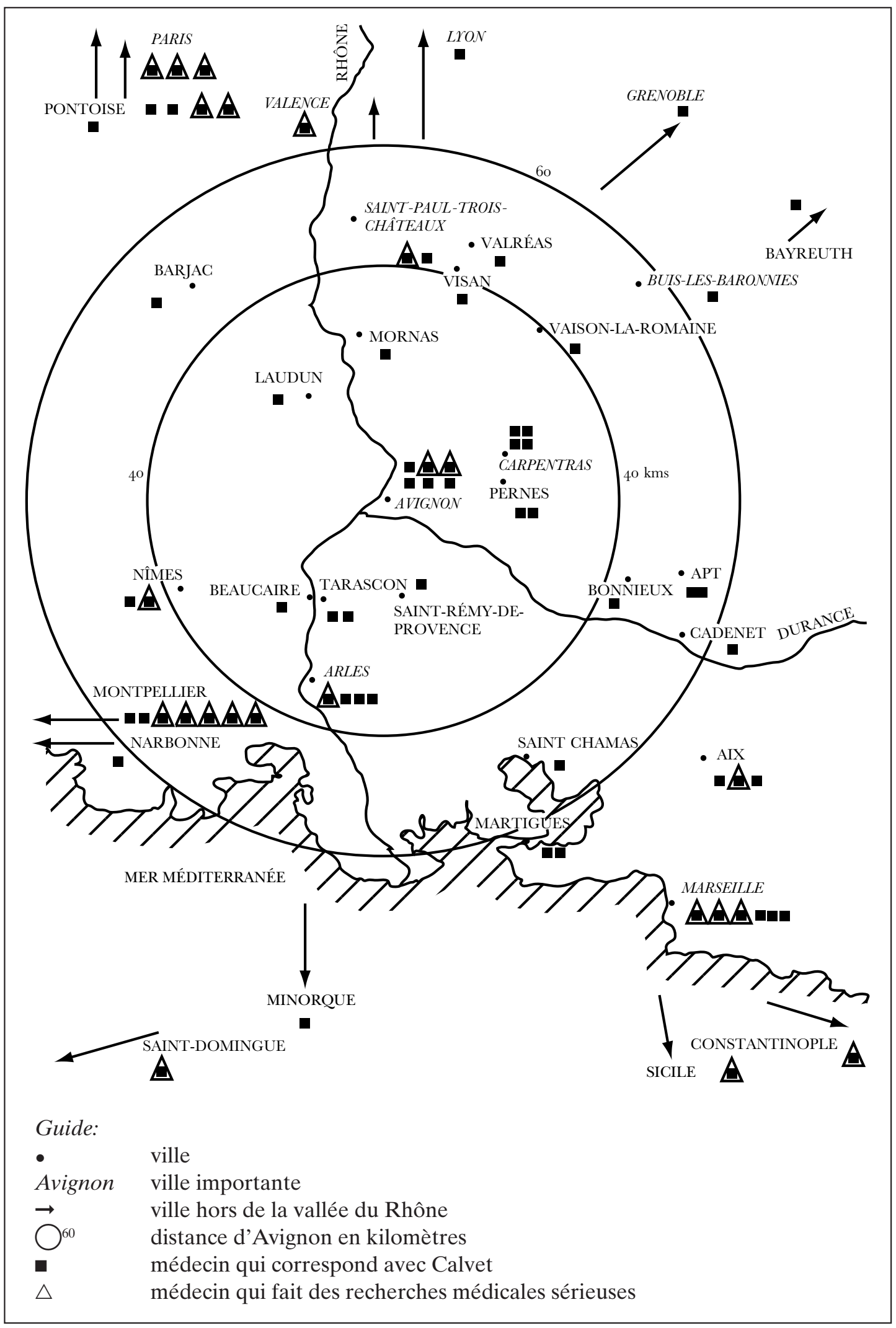

Carte 2. Correspondants médicaux de Calvet (Source: Correspondance survivante de Calvet comme cité sous la note 15). 
Plusieurs médecins à Paris, et même deux correspondants des colonies, comptent parmi leur nombre. Parfois, ses correspondants évoquent dans leurs lettres, au moins en partie, des malades individuels; il en résulte une imbrication entre ce type de correspondance et le premier. Il existe toujours des médecins locaux qui veulent consulter Calvet de temps en temps sur un malade privé, alors que Calvet lui-même envoie des lettres aux médecins les mieux connus de Montpellier au sujet de cas désespérés. En janvier 1780, par exemple, il introduit la marquise de Caumont, une Avignonnaise qui mourra après avoir subi deux opérations pour une tumeur au sein, à François Bourguignon de Lamure (1717-1787) 22 . De façon générale toutefois, cette correspondance entre Calvet et d'autres médecins ne concerne pas des malades spécifiques, mais plutôt la science médicale ou le métier du médecin.

Les lettres abordent en particulier divers aspects de la physiologie ou de l'hygiène, les nouveautés thérapeutiques, les maladies dominantes, les publications récentes, les prix académiques, la difficulté de gagner sa vie comme médecin. Elles nous donnent ainsi une bonne idée des intérêts médicaux de Calvet. Il est clair que ce dernier n'était pas anatomiste. Quoiqu'il possédât dans sa bibliothèque des textes fondamentaux, comme le Anatomia corporum humanorum (édition de 1750) du hollandais Willem Cowper (1666-1709) et le De sedibus et causis morborum per anatomen indagatis (1761) de Giovanni-Batista Morgagni (1682-1771), et quoiqu'il enseignât l'anatomie en 1753/54 à l'Université d'Avignon, ses correspondants médicaux font rarement mention de ce domaine ${ }^{23}$. Seul Tournatoris, un médecin d'Aix, essaie de l'engager dans une correspondance anatomique, en lui offrant d'échanger des spécimens, mais c'est une correspondance qui ne dure que l'espace de deux lettres ${ }^{24}$. De l'autre côté, Calvet est fasciné par le problème de l'inoculation. Il se déclare en faveur de cette pratique au début des années soixante et suit de près la bataille entre ses promoteurs et ses adversaires à Paris. Son espion dans la capitale à la mi-décennie, alors que le débat est particulièrement vif, est un ancien étudiant de médecine d'Avignon, devenu plus tard son collègue dans cette même ville, Isidore-Dominique Vicary. Le 11 octobre 1765, ce dernier lui envoie une lettre relatant un article hostile récemment paru dans la Gazette littéraire. L'année suivante, Vicary envoie à Calvet une copie d'une petite œuvre défendant l'inoculation et écrite par l'anatomiste Antoine Petit (1718-1794), qui avait été professeur parisien

22 BMA Ms 5619, «Collections de consultations», sans feuille: numéro 32 de la collection (numérotation L. B.).

23 BMA Ms 2346, catalogue de la bibliothèque de Calvet, numéros 316 et 411; Brockliss 2002, 28.

24 BMA Ms 2353, fos 346-349: lettres du 11 novembre 1767 et 23 février 1768. 
de l'Avignonnais quinze ans auparavant ${ }^{25}$. La correspondance médicale révèle en outre que Calvet continue à promouvoir l'inoculation au début du $\mathrm{XIX}^{\mathrm{e}}$ siècle face à la campagne en faveur de la vaccination. Dans plusieurs lettres datant des années 1810-1813 adressées à un médecin de Carpentras, Rollandes, Calvet attaque la nouvelle pratique comme dangereuse ${ }^{26}$.

Il existe plusieurs médecins avec lesquels Calvet entretient une correspondance médicale pendant de nombreuses années. Un d'eux est un Sicilien, Joseph Micciari, exerçant la médecine à Messine ${ }^{27}$. La correspondance commence en 1761, après que Micciari eut envoyé le fils du marquis de Solyma à Avignon afin de trouver un remède pour sa maladie des yeux auprès de l'éminent chirurgien-oculiste Pierre-François-Bénézet Pamard (1728$1793)^{28}$. Pendant la décennie qui suit, Calvet aide le Sicilien à acquérir une bonne connaissance de la littérature médicale récente de l'Europe du Nord en lui suggérant les meilleurs titres et en lui offrant de les lui acheter. En revanche, quand le médecin italien demande à Calvet son aide pour faire publier à Avignon son propre livre sur un sujet médical, celui-ci le renvoie en prétendant que les imprimeurs de sa ville natale ne comprennent pas le latin ${ }^{29}$. A cet égard, il se montre plus prêt à aider un autre médecin, François Paul (1731-1774), de Saint-Chamas près de Martigues, qui se spécialise dans la traduction du latin en français de livres médicaux récents, comme la Chirurgie de l'allemand Lorenz Heister (1683-1748), publiée en 1770, ou les Commentaires de Gérard Van Swieten (1700-1772) sur les Aphorismes de Boerhaave, publiés en 1759,1760 et $1766^{30}$.

Trois de ces correspondances médicales sont d'une importance particulière. Claude-François Achard (1751-1809) de Marseille était un élève de Calvet à Avignon. Après avoir obtenu son doctorat, il s'établit dans un petit bourg de Provence, avant de s'installer dans sa ville natale, où il devient

25 BMA Ms 3050, fos 783-786: lettres du 11 octobre 1765 et 7 août 1766. Comme Calvet, Vicary résida à Paris après ses études médicales pendant plusieurs années. Antoine Petit était professeur au Jardin du roi qui donnait des leçons privées à son propre amphithéâtre. C'était un professeur renommé et tous les visiteurs médicaux à la capitale au mi-siècle suivaient son cours.

26 BMA Ms 1254. Calvet ne possédait pas le texte bien connu de Tissot, L'Inoculation justifiée, imprimé pour la première fois à Lausanne en 1754.

27 Rien n'est connu de ce médecin.

28 Pamard perfectionna le technique de guérir la cataracte par extraction: Brockliss 2002, 138.

29 BMA Ms 2344, fos 313sq.: Calvet à Micciari, 28 septembre et 28 octobre 1764 (copies). Son livre, intitulé De signis morbi proxime futuri, ne fut jamais publié. Avignon, ville papale hors de la juridiction du roi de France, était un important centre de publication au XVIII ${ }^{\mathrm{e}}$ siècle: voir Moulinas 1974.

30 Pour le livre de Heister et son supplément, Calvet s'occupa de faciliter les négociations avec la maison d'édition et de corriger les épreuves: voir BMA Ms 2353, fos 149sq.: Paul à Calvet, lettres du 29 décembre 1768 au 23 décembre 1772. 
secrétaire à l'académie puis, pendant la Révolution, conservateur de la nouvelle bibliothèque municipale ${ }^{31}$. Achard entretient une correspondance avec le médecin avignonnais pendant quarante ans et, au début de sa carrière surtout, il discute continuellement avec son ancien professeur de ses soucis professionnels. Dans une lettre, il se plaint de l'insolence des chirurgiens; dans une autre, il accuse ses collègues à Marseille d'ignorer l'avis de Thomas Sydenham sur la valeur de l'exercice; dans une troisième, il recherche l'avis de Calvet sur le système vitaliste de Paul-Joseph Barthez (1734-1806), professeur à Montpellier ${ }^{32}$. Leur relations demeurèrent toujours étroites, agrémentées de visites mutuelles. De fait, pendant les premières années de la Révolution, le célibataire Calvet, désormais âgé, alla habiter chez Achard et sa jeune famille à Marseille pendant plusieurs mois ${ }^{33}$. Tout aussi rapproché était le rapport entre Calvet et un second correspondant important, JeanGabriel Niel (1774-1853), qui devint vers la fin de sa vie le médecin de l'écrivain Châteaubriand. Niel était le fils et cousin d'imprimeurs avignonnais. Comme Calvet était un habitué de la maison Niel, il connaissait Jean-Gabriel depuis son enfance et était heureux de l'aider à s'établir comme médecin après la Révolution ${ }^{34}$. Leur correspondance révèle que le jeune médecin attendait beaucoup de l'ami de sa famille. Il s'installa initialement dans la petite ville de Saint-Paul-Trois-Châteaux en 1796, mais, après cinq années, décida de partir à cause du manque de clientèle. Il demanda aussitôt à Calvet de l'aider à trouver une situation plus lucrative, et réussit finalement à déménager à Marseille, où les autres médecins lui firent bon accueil grâce aux lettres d'introduction écrites par Calvet en sa faveur ${ }^{35}$. Une fois installé, Niel devint de plus en plus positif; ses lettres contenaient en général des rapports sur les idées médicales en vogue à la cité et informaient Calvet de son progrès solide comme médecin ${ }^{36}$.

Par contraste, le troisième correspondant, Félix Vicq d'Azyr (1748-1794), ne devint jamais un ami. Médecin parisien et professeur d'anatomie à l'école

31 Nulle étude n'existe sur la vie d'Achard, quoiqu'il ait été un personnage important dans la vie culturelle de Marseille. La bibliothèque municipale de la cité se construisit grâce aux livres confisqués de l'Eglise et aux émigrés. Pour la nomination d'Achard en tant que bibliothécaire le 30 août 1793, voir Brockliss 2002,353n.

32 BMA Ms 2361, fos 11-13, 39sq., 91sq.: Achard à Calvet 24 avril 1772, 8 janvier 1776, 15 septembre 1779. Calvet n'était pas influencé par le vitalisme: Brockliss 2002,152sq.

33 Brockliss 2002,33sq.

34 Jean-Joseph Niel était le seul imprimeur d'Avignon. Calvet le décrit comme un ami: Brockliss 2002, 69.

35 BMA Ms 5618, fos 206-214,319-368: Niel à Calvet, lettres 5 floréal an IX-21 brumaire an XII (sans ordre chronologique).

36 Par exemple, BMA Ms 5618, fos 223sq.: Niel à Calvet 1 août 1805: sur l'influence à Marseille de l'écossais John Brown (1735-1788) et d'autres auteurs de systèmes médicaux. 
vétérinaire à Alfort près de la capitale, il devint secrétaire de la Société royale de médecine en 1776. Cette nouvelle institution était dédiée principalement à la collection de statistiques médicales dans l'espoir de mieux contrôler les épidémies humaines et animales auxquelles la France du XVIII ${ }^{e}$ siècle était en proie ${ }^{37}$. A cet effet, la Société avait besoin de correspondants provinciaux qui établissent chaque année un rapport sur l'histoire des épidémies à leur région. Calvet, en tant que médecin le mieux connu au Comtat Venaissin, fut nommé dès le début membre correspondant de la Société et maintint une correspondance avec le secrétaire de cette dernière jusqu'à la Révolution. A l'évidence, une telle correspondance était purement formelle: de temps en temps, Calvet écrivait à Vicq en lui racontant des choses médicales intéressantes, et de temps en temps Vicq répondait, afin d'encourager son correspondant et de lui envoyer de nouvelles requêtes. Il est clair que Calvet, qui s'en plaignait souvent, n'aimait pas cette obligation ${ }^{38}$.

Mais la correspondance avec Vicq d'Azyr n'en demeure pas moins d'un grand intérêt. Tout d'abord, les lettres des deux médecins sont conservées: celles de Calvet lui-même à la bibliothèque de l'Académie de médecine à Paris et en tant que copies prises par l'auteur à Avignon, où se trouvent également celles de son correspondant. De plus, les lettres de Calvet sont particulièrement riches en informations médicales. Elles renseignent sur les épidémies à Avignon dans les dernières années de l'Ancien Régime et la façon dont on essaie de les traiter ${ }^{39}$. Elles révèlent le succès de Calvet avec l'inoculation, ses expériences ambiguës avec l'électrothérapie pour soigner la paraplégie ${ }^{40}$. Et surtout, elles nous informent sur les activités des guérisseurs sans licence qui exerçaient la médecine à Avignon à cette date et les remèdes qu'ils vendaient. La Société royale de médecine étendait sa compétence jusqu'à comprendre l'élimination des charlatans qui, selon Vicq, étaient responsables de la grande mortalité des maladies épidémiques en France. Calvet souscrivait totalement à ce jugement. Médecin de plus en plus soupçonneux envers les thérapies interventionnistes, il haïssait les charlatans qui, disait-il, étaient partout à Avignon, notamment un forgeron du nom de Buisson $^{41}$. Au fond, croyait-il, le problème résidait dans les malades insistant pour que leurs médecins prescrivent les remèdes désirés sans égard envers

37 Hannaway 1974.

38 Par exemple, BMA Ms 2345, fos 9sq.: Calvet à Vicq, 2 septembre 1778 (copie).

39 Par exemple, BMA Ms 2345, fos 20-23: Calvet à Vicq 26 octobre 1780, sur une fièvre guérie par la quinine (copie).

40 Par exemple, BMA Ms 2345, fos 5-9, 12-15: Calvet à Vicq, $1^{\text {er }}$ juillet 1778, 6 juillet 1779 (copies).

41 BMA Ms 2345, fo 37: Calvet à Vicq 8 novembre 1786. Sur le problème croissant des empiriques au XVIII' siècle, voir Brockliss/Jones 1997, chap. 10. 
leur valeur médicale. Il cite par exemple dans une lettre de 1779 le cas d'une malade qui lui avait demandé de prendre le Rob Laffecteur pour guérir d'une maladie vénérienne, quoique ce remède - un cordial végétal récemment inventé à Paris - fût empirique et douteux. Calvet le lui interdit, mais la malade insiste, avale quatre bouteilles du remède sans résultat, et reprend encore une autre bouteille contre l'avis du professionnel ${ }^{42}$.

\section{La correspondance entre le médecin et les autres érudits}

Une correspondance entre deux médecins ne porte pas nécessairement sur les seuls sujets médicaux. Plusieurs des correspondants de Calvet partageaient ses intérêts intellectuels plus larges et étaient prêts à développer ses collections. Achard et Niel étaient également des antiquaires, qui faisaient souvent référence à leur passion dans leurs lettres. En 1802 par exemple, Niel écrit à Calvet qu'il est occupé à rédiger une communication sur la nature et l'utilité de l'étude des antiquités qu'il a l'intention de présenter au concours de l'Institut ${ }^{43}$. Malheureusement, des livres lui manquent et il demande à Calvet de lui envoyer des notes sur les tomes dont il a besoin ${ }^{44}$. De fait, il arrive qu'un correspondant, même s'il s'agit d'un médecin important, n'évoque jamais de son métier dans ses lettres à Calvet. Il s'agit de Roustan de Nîmes, médecin et naturaliste qui maintint une correspondance avec le médecin d'Avignon pendant les années soixante. Toutes ses lettres concernent sa collection et l'échange des spécimens avec son $\mathrm{ami}^{45}$.

Par conséquent, de même qu'on ne peut pas séparer de façon absolue une correspondance entre médecins d'une correspondance entre un médecin et ses malades, de même, on ne peut pas séparer nettement une correspondance strictement médicale d'une correspondance d'un médecin avec la République des Lettres plus large, bien qu'une division effective demeure. En plus de ses consultations médicales, Calvet entra en correspondance durant sa vie avec au moins 350 personnes, dont seulement 68 étaient des médecins. Quoique plusieurs de ses correspondants non médicaux fussent des anciens

42 BMA Ms 2345, fos 15-17: Calvet à Vicq, 18 octobre 1779. Sur le Rob, voir Bouvet 1923.

43 L'Institut, nouvelle académie des sciences et lettres de Paris, remplace les académies d'Ancien Régime en 1795.

44 BMA Ms 5618, fos 321-323, 345sq., 365sq.: Niel à Calvet, 12 décembre 1801, 24 juin et 5 juillet 1802.

45 BMA Ms 2353, fos 240-329. Roustan obtint son diplôme de doctorat à Montpellier en 1746. Pour une description de sa belle collection d'histoire naturelle, voir Desallier d'Argenville 1780, I, 295sq. 
malades et d'autres discutassent de leurs maladies et de celles de leur famille dans leurs lettres, leur but principal en écrivant à l'érudit avignonnais était de parler avec lui des antiquités, de l'histoire naturelle, ou de la littérature grecque et romaine. Ils (car ce sont tous des hommes) écrivaient à Calvet, non pas parce qu'il était médecin, mais parce qu'il était un érudit bien connu dans la vallée du Rhône, avec lequel l'on pouvait partager un intérêt commun ${ }^{46}$.

Autrement dit, la plus grande partie de la correspondance de Calvet ne concerne pas les affaires médicales, et le statut intellectuel qu'il acquiert dans le monde de son époque ne dérive pas de son métier mais de ses passe-temps. Cela devient évident lorsqu'on centre son attention sur ses correspondants les plus importants. Le diagramme 1 montre que Calvet n'avait que trentedeux correspondants permanents, c'est-à-dire des correspondants lui ayant envoyé plus de vingt lettres et ayant entretenu avec lui une correspondance continue pendant plusieurs années, parfois leur vie durant. De fait, à n'importe quel moment, Calvet avait au maximum dix-sept correspondants réguliers seulement, ce qui demeure un nombre assez important si l'on rappelle qu'une lettre comportait habituellement quatre pages pleines de renseignements techniques. Comme le montre la carte 3 , la plupart de ses correspondants permanents habitaient eux aussi dans la vallée du Rhône, hormis quatre Parisiens. Calvet, même comme érudit, demeurait provincial. Il n'avait pas beaucoup de contacts intellectuels ou médicaux hors du Midi. Par exemple, son seul correspondant suisse dans la République des Lettres était le naturaliste Elie Bertrand (1713-1797), pasteur de l'église française de Berne, qui lui demanda en 1765 de l'aider à rééditer une collection de ses dissertations à Avignon ${ }^{47}$.

Les membres de ce petit réseau permanent comprennent six membres de la noblesse d'épée (cinq anciens militaires), sept avocats ou officiers (plusieurs aussi nobles), six médecins (dont un, Roustan, ne parlait pas de son métier), un marchand, et douze gens d'Eglise (dont un était un évêque). Il s'agit à l'évidence d'un réseau de gens professionnels et instruits, où les médecins constituent une minoritét ${ }^{48}$. De plus, c'est aussi un réseau dont la plupart des membres sont des amis mutuels, comme le montre le dia-

46 Pour cette correspondance, voir Brockliss 2002, chap. 2. Calvet eut des rapports aimables avec plusieurs femmes, mais avec une seule qui fût également érudite: Isabella Byron (17211795), Comtesse de Carlisle, qui habitait à Avignon dans les années 1770: Brockliss 2002, 31sq., 113.

47 BMA Ms 2358, fos 3-25: Bertrand à Calvet, lettres du 12 avril 1765 au 12 octobre 1767. Il s'agit de son Recueil de divers traités sur l'histoire de la terre et des fossiles (Avignon 1766).

48 Brockliss 2002, 79-84. 
gramme 2. Chose importante, seul le médecin Roustan appartient à ce réseau interactif dont les autres médecins sont exclus. Le vrai réseau de correspondance de Calvet est clairement un petit cercle d'antiquaires et naturalistes, pour qui la médecine n'a qu'un intérêt marginal. Chose importante encore, ce cercle est organisé. Il n'a pas d'attachement institutionnel ni de profil public; la moitié du groupe ne publient jamais rien, ni n'appartiennent à une quelconque académie, même en tant que membres correspondants. Ce cercle est donc un club privé, avec de nombreux contacts dans le monde républicain plus large, bien entendu, mais essentiellement autonome quant à ses activités intellectuelles mutuelles.

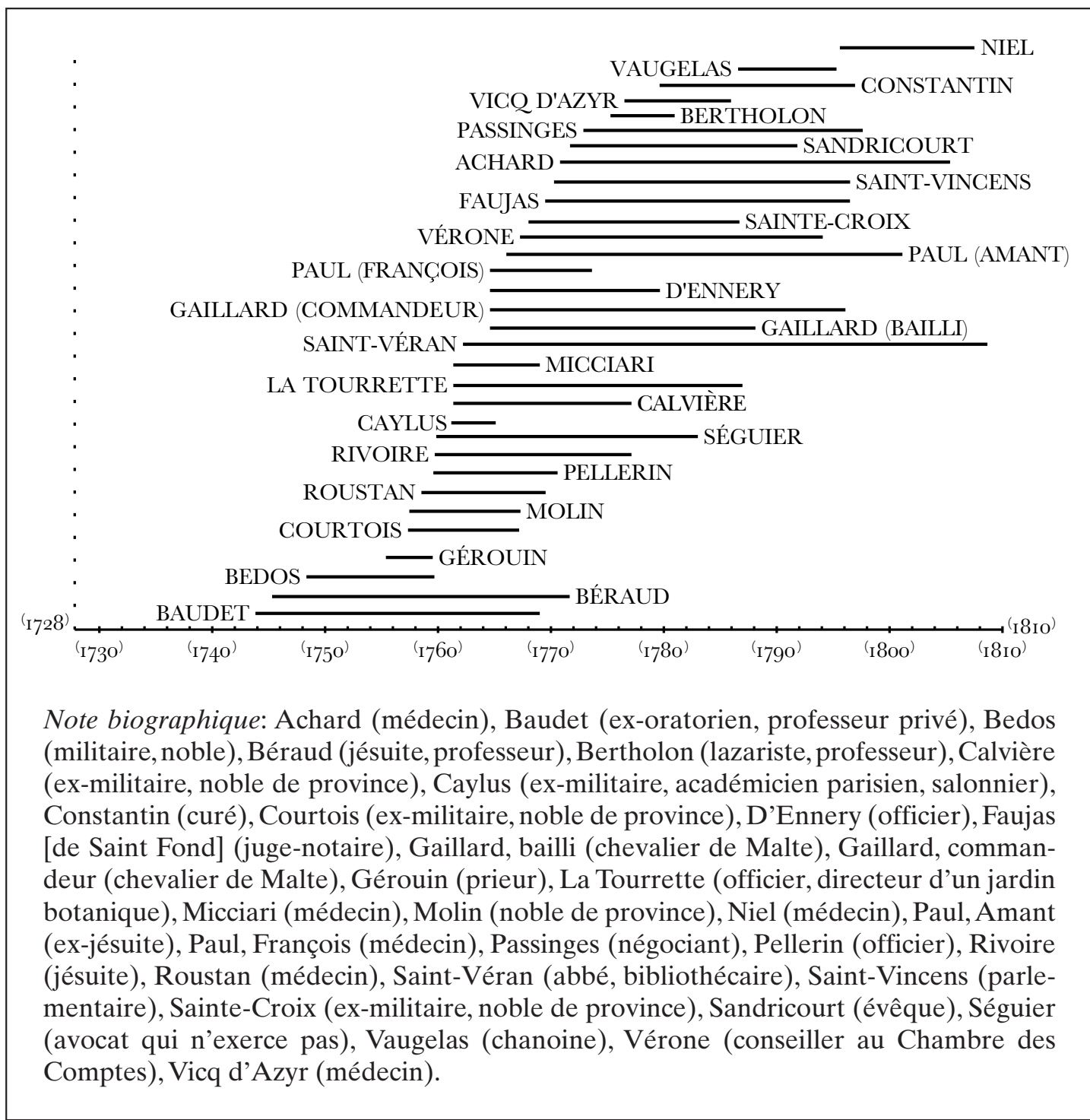

Diagramme 1. Correspondants permanent de Calvet: leurs noms et la période de leur échange épistolaire (Source: Correspondance survivante de Calvet comme cité sous la note 15$)$. 


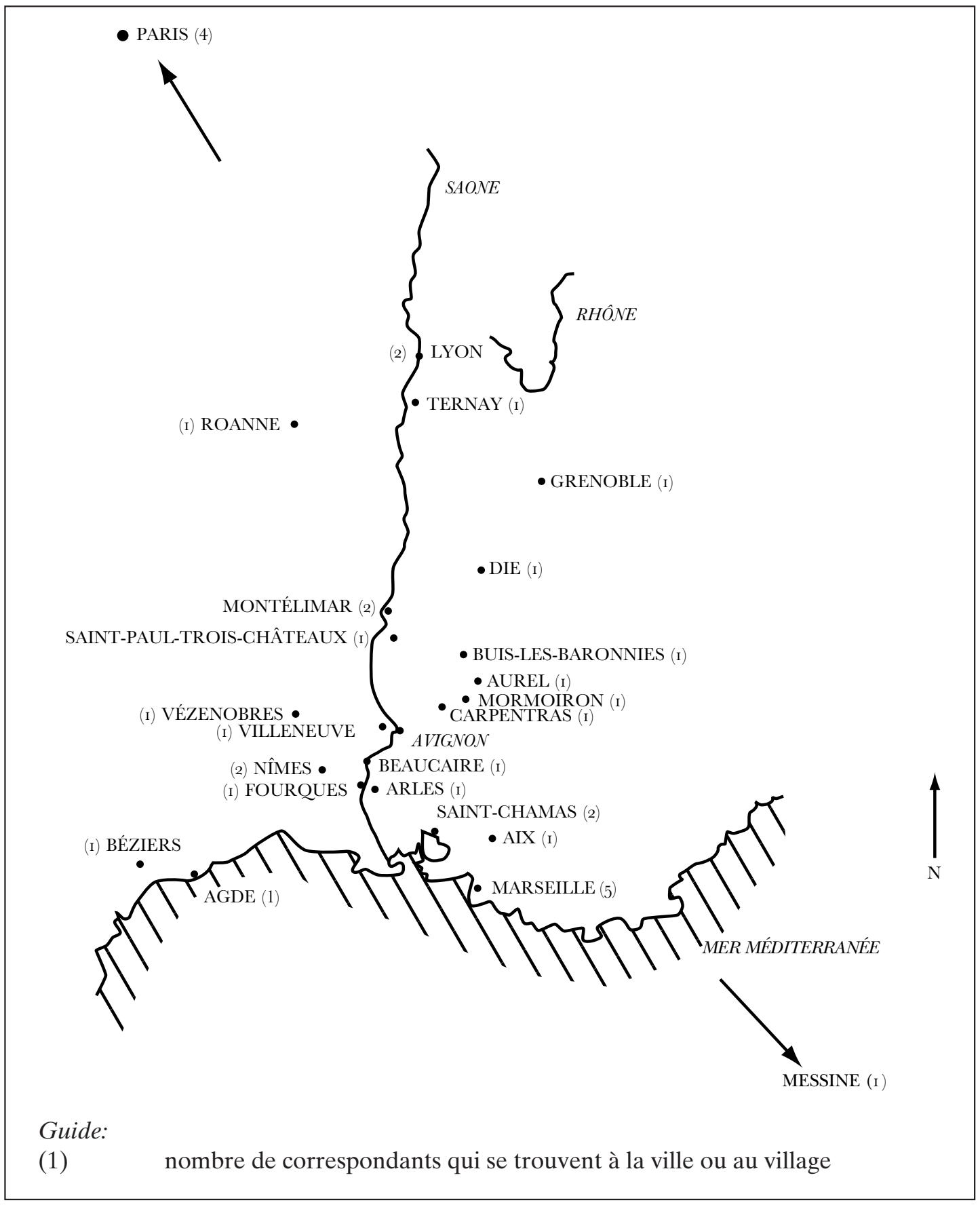

Carte 3: Résidence normale des correspondants les plus réguliers de Calvet (Source: Correspondance survivante de Calvet comme cité sous la note 15). 


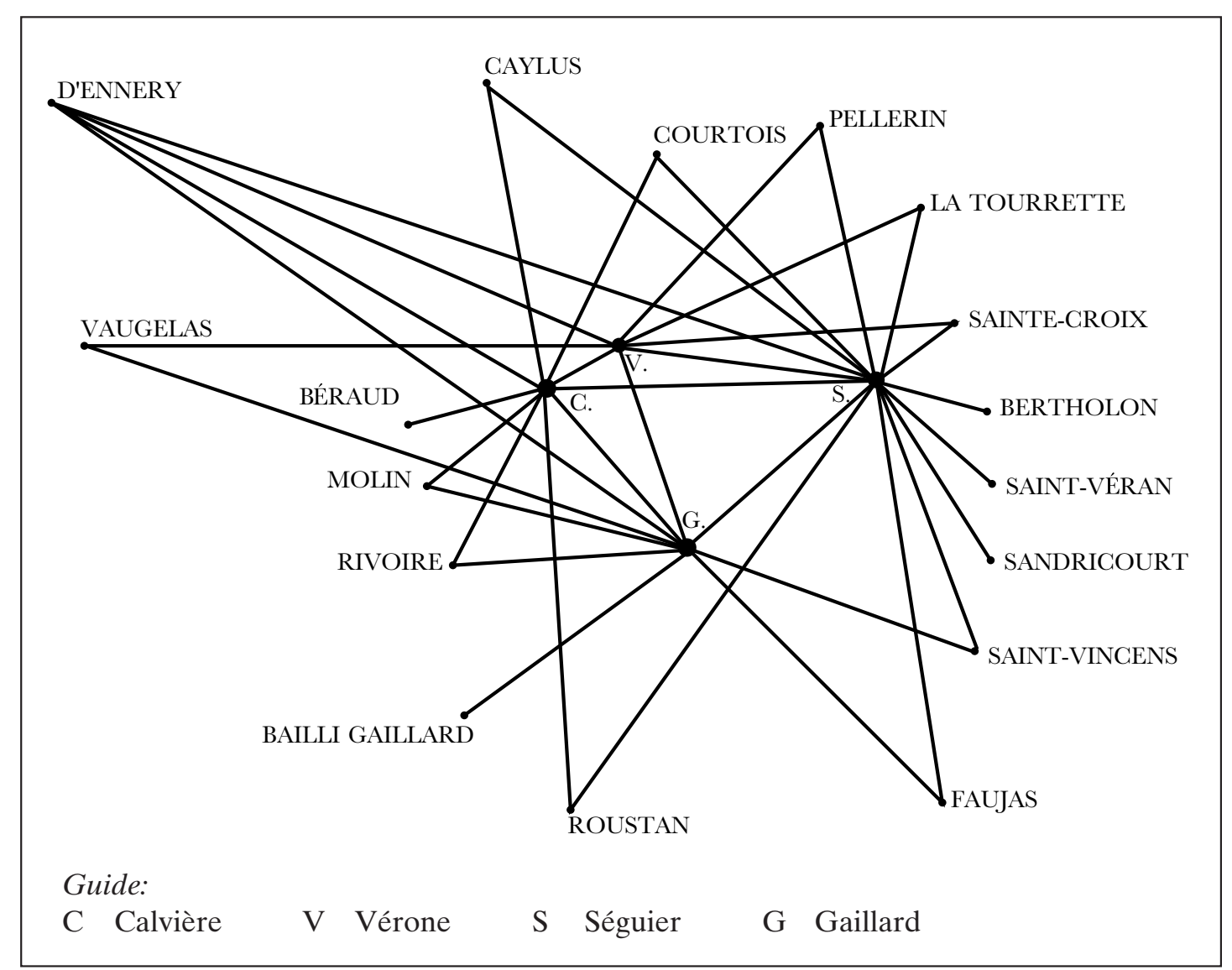

Diagramme 2. Rapports entre les correspondants permanents de Calvet. Le diagramme révèle les contacts entre quatre des correspondants de Calvet - Calvière, le commandeur Gaillard, Séguier et Vérone - et d'autres membres de son cercle intime (Source: En particulier BMA Mss 2355, 2356, 2365, 4447, 5833; Bibliothèque Nîmes Ms 140: correspondance entre Calvet et Calvière, Séguier, Gaillard et Vérone). Les lettres font continuellement référence aux contacts que les quatre possédaient avec d'autres érudits du petit cercle.

\section{Le médecin et l'érudit}

Faut-il conclure qu'il n'y a presque aucune connexion entre Calvet le médecin et Calvet l'érudit? Si nous en croyons Calvet lui-même, assurément. Son intérêt pour les antiquités en particulier commença, semble-t-il, alors qu'il était élève des jésuites à Lyon, où il tomba sous l'influence du Père Laurent Béraud (1702-1777), conservateur du cabinet de collège. Plus tard, en étudiant la médecine à l'Université d'Avignon, cet intérêt fut ravivé par un certain Bedos, officier d'armée au fort de Saint-André à Villeneuve-lès-Avignon, puis affermi définitivement lors de son séjour de deux années à Paris. Autrement dit, Calvet devint un collectionneur passionné avant de commencer à 
exercer la médecine à sa ville natale ${ }^{49}$. Et il était toujours irrité par le fait de devoir passer la plupart du jour à pratiquer son métier au lieu de s'immerger dans ses autres intérêts intellectuels. Dans ses lettres à ses amis intellectuels, il se plaint fréquemment de sa fortune et envie les érudits qui peuvent passer l'entier de leurs journées comme antiquaires ou naturalistes ${ }^{50}$.

Il n'y a aucun doute que Calvet était très occupé comme médecin. La plupart de ses correspondants permanents étaient à la retraite ou exerçaient un métier exigeant peu d'effort. Avant sa mort en 1784 par exemple, l'antiquaire et naturaliste le plus éminent de la vallée du Rhône, Jean-François Séguier de Nîmes (né en 1703) était homme de loisir. Avocat, il n'avait jamais exercé et avait passé sa vie adulte jusqu'à 1755 comme secrétaire de l'érudit italien Francesco-Scipione Maffei (1675-1755) à Vérone ${ }^{51}$. Par contraste, Calvet dut travailler comme médecin jusqu'à l'âge de soixante-quatorze ans. En plus du nombre croissant de malades privés qu'il devait visiter ou qui venaient le consulter chez lui, il avait des responsabilités publiques. Pendant presque vingt ans, il fut professeur à la faculté d'Avignon, et sa vie durant, il eut la charge de médecin de l'hôpital Sainte-Marthe, où, six mois par an, il devait visiter les malades deux fois par jour. De ce fait, le gouverneur papal ou la municipalité lui commandaient régulièrement des rapports sur les épidémies ${ }^{52}$.

Nécessairement donc, Calvet ne pouvait s'adonner à ses passe-temps intellectuels que le soir, quand il n'était pas interrompu par des malades ou des amis de la ville. Mais, n'étant pas riche, il n'avait guère le choix. Son père lui avait légué un patrimoine rapportant peut-être deux mille livres par an: une telle somme était presque suffisante pour qu'un célibataire (et Calvet ne maria jamais) vive à son aise, mais non pour constituer une belle collection et tenir un profil public élevé en tant qu'antiquaire ou naturaliste. Si l'on voulait construire une bibliothèque savante, il fallait beaucoup d'argent. Enfin, grâce à son métier (dont il devait tirer quelque quatre mille livres par an) et à l'intérêt sur ses investissements (il épargnait autant que possible et prêtait de l'argent aux individus et aux municipalités locales), il possédait un revenu de treize mille livres à la veille de la Révolution ${ }^{53}$. Par conséquent, il devint

49 Brockliss 2002, 195. Béraud était aussi un physicien à qui l'Académie de Bordeaux octroya un prix pour un essai sur l'électricité et le magnétisme.

50 Par exemple, BMA Ms 4614, sans foliatage: brouillon d'une lettre à Jules-François-Paul Fauris de Saint-Vincens (1718-1798), parlementaire d'Aix, 26 janvier 1772.

51 Mosele 1981, Séguier (à paraître). Pour sa correspondance, elle aussi très vaste, voir Roche 1987, chap. 11.

52 Selon son livre des comptes, il continuait à recevoir un revenu de son métier en 1803: BMA Ms $5622, \mathrm{f}^{\text {os }} 63$ sq.

53 Brockliss 2002, 52-62. 
assez riche pour jouer le rôle qu'il désirait dans la République des Lettres, même s'il ne put jamais concurrencer les milords anglais dans l'achat des plus belles antiquités italiennes - les urnes grecques et étrusques, les grandes statues romaines, etc. ${ }^{54}$ Son métier ne cessa toutefois d'occuper la première place dans sa vie. Sans un Calvet médecin, pas de Calvet antiquaire et naturaliste. Le métier contraignit toujours l'érudit.

L'attitude affichée de Calvet envers son métier paraît toutefois trop dure, car ce dernier l'aida beaucoup à construire ses collections. Il pouvait acheter de temps en temps des médailles, des morceaux d'antiquités ou des minéraux (il s'intéressait en particulier au règne minéral et aux fossiles), offerts par des brocanteurs ou des héritiers pressés de se débarrasser du bric-à-brac de leurs parents ${ }^{55}$. En général, cependant, Calvet évitait le marché. Il ne dépensait pas beaucoup pour ses collections: environ 9500 livres pour ses médailles et antiquités, 250 livres pour sa collection d'histoire naturelle ${ }^{56}$. Il en constitua l'essentiel grâce à des échanges, des dons et des découvertes locales qu'il réussit à acquérir. Et dans ces situations, son métier était d'une importance incalculable. Tout d'abord, sa clientèle qui connaissait ses intérêts intellectuels était heureuse de lui donner des cadeaux en reconnaissance de son aide professionnelle. Les copies de deux grandes statues antiques, que Calvet conservait dans son vestibule avaient pu être emportées de Rome grâce à un don d'un voyageur, Monsieur Arnoux, habitant à Rochegude, une petite ville près d'Alès ${ }^{57}$. Fait plus important encore, Calvet utilisait ses anciens élèves médecins comme agents de terrain. Puisqu'ils exerçaient leur métier pour la plupart dans les petits bourgs de Provence et du Dauphiné, ils se trouvaient dans une position parfaite pour l'avertir qu'un paysan avait déterré une trouvaille et pour tenter de l'obtenir pour leur ancien professeur. Le 10 avril 1790 par exemple, Calvet reçut une lettre du médecin Jacques-Nicolas Esperandieu de Buis-les-Baronnies l'informant qu'un paysan venait de découvrir des objets antiques en bronze d'un poids énorme: 30 livres. Mais ce paysan refusait de les lui vendre pour leur seule valeur métallique. Que faire? Esperandieu attendait les instructions de son maitre ${ }^{58}$. Calvet croyait aussi que les médecins (ou plutôt ses amis médecins) étaient ses meilleurs agents. En août

54 En Italie, dans la première moitié des années 1770, James Hugh Smith Barry dépensa 125000 livres pour les grandes statues: voir Vaughan 1987.

55 Il obtint de cette façon les médailles de son ami le Marquis de Calvière (1693-1777), vendues à bon marché par son fils, le comte, qui avait besoin d'argent: BMA Ms 2355,

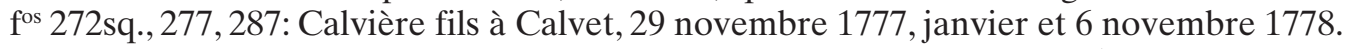

56 BMA Mss 5621sq.: ses livres des comptes couvrent la période 1767-1808 (il catalogue chaque mois ses dépenses).

57 BMA Ms 2346, fo 188r-v: inventaire de la collection d'antiquités de Calvet.

58 BMA Ms 5168, fos 94 sq. 
1769, Séguier lui écrivit en demandant son aide pour trouver des semences d'une plante qui se trouvait autour de Bollène, une ville au bord du Rhône au nord d'Avignon. Séguier suggérait que Calvet entre en contact avec des moines locaux, mais ce dernier préféra utiliser un médecin de la place, son ancien élève Joseph-Philippe Martinel ${ }^{59}$. Autrement dit, non seulement pas de Calvet collectionneur et érudit sans un Calvet médecin, mais aussi pas de riches collections sans clients ni élèves médecins.

\section{Un citoyen-médecin typique?}

En conclusion, peut-on dire que Calvet était représentatif des gens de l'art jouant un rôle actif dans la République des Lettres de la deuxième moitié du dix-huitième siècle? Ou était-il simplement un cas solitaire, dont la vie est intéressante mais sans signification générale? Il est évident que Calvet n'est pas typique des grands citoyens médicaux. Ni le Calvet médecin ni le Calvet savant ne sont comparables au suisse Albert de Haller, cet esprit original et créatif dont la correspondance reflète la réputation internationale ${ }^{60}$. Et on doit avouer aussi qu'en l'état présent des recherches, il est également impossible de juger vraiment si Calvet était représentatif des citoyens-médecins modestes. Pour se lancer dans des études comparatives, il nous faut attendre la découverte dans des archives françaises d'autres correspondances médicales aussi riches que celle de Calvet. Néanmoins, les renseignements existant sur la vie intellectuelle de deux autres citoyens-médecins du Midi avant et pendant l'époque révolutionnaire suggèrent que Calvet comme savant était assez typique des médecins de talent modeste dans sa propre région.

Pierre-Joseph Amoreux (1741-1824) et Dominique Bouchet (dates de naissance et de mort inconnues) étaient tous deux médecins diplômés de Montpellier et de la même génération qu'Achard et Niel, les deux correspondants médicaux les plus chers de notre érudit avignonnais. Né à Beaucaire, Amoreux était le fils d'un médecin naturaliste, Guillaume (1714-1790), qui déménagea à Montpellier pendant que Pierre-Joseph y étudiait ${ }^{61}$. Né, comme Calvet à Avignon, Bouchet était le fils d'un négociant, membre-fondateur de l'Athenée de Vaucluse devenue plus tard l'Académie, au début de l'époque

59 Bibliothèque Municipale de Nîmes, Ms 140, fo 120: Calvet à Séguier, 9 août 1769.

60 Haller avait 1200 correspondants, dont 91 résidant en France: voir Boschung 2002.

61 Pour sa famille et sa jeunesse, voir BMA Ms 1269, p. 2-33: autobiographie de Pierre-Joseph Amoreux. Avec James Livesey, je prépare une édition électronique de ce manuscrit. Amoreux père employa Calvet pour surveiller la publication de la thèse de doctorat de son fils en 1762: voir BMA Ms 2358, fo 1: Amoreux père à Calvet, 20 avril 1762. 
napoléonienne ${ }^{62}$. Accomplissant ses études dans la nouvelle école de santé de Montpellier établie en 1794, il appartenait non seulement à une génération beaucoup plus jeune que l'érudit avignonnais, mais aussi à une époque différente ${ }^{63}$. Tous les deux étaient des botanistes acharnés; de plus, Amoreux s'intéressait à l'agronomie et la médecine vétérinaire ${ }^{64}$. A cet égard, ils se distinguent entièrement de Calvet. Ce dernier était un antiquaire aussi bien qu'un naturaliste; et comme naturaliste, nous l'avons déjà mentionné, il s'intéressait surtout aux fossiles et minéraux. Quoique linnéen, il ne portait pas beaucoup d'intérêt à la botanique et ne possédait pas luimême de jardin, et même s'il était propriétaire, il négligeait totalement l'agronomie $^{65}$.

Comme Calvet, Amoreux n'avait pas de revenu privé. Il était donc contraint, avant la Révolution, d'exercer son métier, en dépit du fait qu'il le trouvât un peu ennuyeux. Il était aussi premier bibliothécaire de la bibliothèque de l'ancienne faculté de médecine de Montpellier fondée par le professeur de médecine Henri Haguenot en $1768^{66}$. Bouchet en revanche avait davantage de chance. Avant même d'obtenir son diplôme à Montpellier, il épousa la fille d'un riche négociant de la ville, qui lui apporta 100000 livres comme dot et la promesse de 600000 autres livres. Il put par conséquent passer toute sa vie adulte sans devoir gagner son pain ${ }^{67}$. Tous deux nous ont laissé une collection de 300 lettres adressées à eux et concernant pour la plupart leurs intérêts intellectuels, pour Amoreux pendant les années 1770 et 1780 , pour Bouchet dans le premier quart du XIX ${ }^{\mathrm{e}}$ siècle ${ }^{68}$. Leurs auteurs, comme on peut s'y attendre, étaient des avocats, des abbés et d'autres médecins partageant leur enthousiasme pour la botanique ou l'agronomie. Amoreux avait cinquante-cinq correspondants, Bouchet seulement trente-neuf, mais tous deux, comme Calvet, n'eurent que peu de correspondants vraiment

62 Rien n'est connu de la vie de Bouchet père, sauf son rôle de fondateur de l'Athenée. Au $\mathrm{XVIII}^{\mathrm{e}}$ siècle, Avignon était dépourvue d'académie, bien qu'on en trouvât dans presque chaque ville importante de France.

63 Bouchet fils était connu de Calvet: voir BMA Ms 3051, fo 142: Calvet à Bouchet fils, 8 mars 1806.

64 Il appartenait à ce groupe d'agronomes de Languedoc qu'étudie James Livesey: voir cidessus, note 7.

65 Brockliss 2002, chap. 5.

66 BMA Ms 1269,p. 45,47sq., 83-89: quand son père meurt en 1790, il ne reçoit que 20000 livres. Pour preuve de la valeur de son métier, voir son livre des comptes: Bibliothèque Municipale de Nîmes, Ms 291.

67 BMA Ms 4426, fos 198-214: André Guillaume Calvet ([1761-1825]: un parent distant de l'Avignonnais et chanoine de la cathédrale à Avignon avant la Révolution) à Bouchet père, 8 frimaire et 10 nivôse an 7 .

68 Bibliothèque Nationale Ms français nouvelles acquisitions (désormais BN Ms fr. n.a.), 6570-6572. 
permanents: trois pour Amoreux, six pour Bouchet ${ }^{69}$. Amoreux, comme Calvet encore, trouvait ses correspondants dans le Midi, même si son réseau de naturalistes s'étendait au-delà de la vallée du Rhône en direction de Toulouse. De fait, même sa poignée de correspondants parisiens étaient pour la plupart des gens du Sud, ou des émigrés comme le médecin de cour JosephBarthélemy-François Carrère (1740-1802) de Perpignan, ou encore des visiteurs de la capitale ${ }^{70}$. Amoreux avait un seul correspondant étranger, le médecin Jean-Jacob Dapples de Lausanne (c. 1701-1774) ${ }^{71}$. Bouchet, par contraste, avait un réseau un peu plus étendu qui comprenait un Caennais, des Espagnols, des Allemands et un Suisse, Johann Jakob Römer (17361819), médecin zurichois et rédacteur de plusieurs journaux de botanique ${ }^{72}$.

Quoique Amoreux et Bouchet fussent des botanistes, leurs deux réseaux de correspondance étaient relativement distincts. Il est évident que les bouleversements de l'Ancien Régime causèrent une fracture immense dans le monde botanique du Midi. Le seul correspondant que partageaient les deux érudits était Antoine Gouan (1733-1821), le doyen des botanistes montpelliérain, un homme du monde prérévolutionnaire mais demeurant actif pendant et après la Révolution ${ }^{73}$. D'un autre côté, même si les intérêts intellectuels de Calvet et d'Amoreux étaient différents, ces deux citoyens de la République de l'Ancien Régime avaient plusieurs correspondants en commun. Dans le cas de Vicq d'Azyr, cela reflète le fait qu'Amoreux était aussi membre correspondant de la Société Royale de Médecine, et un membre qui participait davantage aux activités de celle-ci que le médecin avignonnais $^{74}$. Plus généralement toutefois, ce fait souligne que la plupart des citoyens prérévolutionnaires présentaient une grande variété d'intérêts intellectuels et pouvaient appartenir à plusieurs réseaux séparés. Calvet et

69 Chose inattendue, la collection de Bouchet comprend une correspondance permanente avec une Madame Siméon entre 1814 et 1831. Il ne s'agit pas d'une correspondance érudite au sens strict, mais concerne le commerce du vin.

70 BN Ms fr. n.a. 6570, fos 87-102: correspondance de 1775 à 1787. Carrère était originalement professeur de médecine à Perpignan. Il partit pour Paris en 1773 et publia divers traités de thérapeutique.

71 Cinq lettres 1760-1762: BN Ms fr. n.a. 6570, fos 42-51. Dapples visita le Midi en 1760 et aurait pu rencontrer l'étudiant Amoreux à Beaucaire ou à Montpellier. Dapples était un correspondant de Haller.

72 BN Ms fr. n.a. 6572, fos 384-404: douze lettres 1803-1812. La correspondance concerne un échange de plantes. Römer était professeur à l'Institut médico-chirurgical de Zurich, et prépara une édition d'une sélection des comptes-rendus de Haller dans les Göttingische Gelehrte Anzeigen (2 vols, 1789-1791). Je dois ces renseignements à Hubert Steinke.

73 BN Ms fr. n.a. 6570, fos 189-209 (1764-1765, 1775); n.a. 6572, fos 159-204 (c. 1799-1811). Il n'existe toujours pas d'étude de la vie et l'œuvre de Gouan.

74 BN Ms fr. n.a. 657, fos 293-339: Vicq à Amoreux. Amoreux présenta quatre mémoires sur des sujets médicaux à la Société: voir ibid., fos 295sq., 307sq., 317sq., 338sq.: 20 juillet 1781, 8 octobre 1784,18 juillet $1787,1^{\text {er }}$ septembre. 
Amoreux pouvaient partager le même correspondant, mais ils écrivaient rarement à leur ami mutuel sur même sujet. Prenons l'exemple du nîmois Séguier, un correspondant cher à l'un comme à l'autre ${ }^{75}$. Séguier était un grand antiquaire qui possédait une belle collection d'épigraphes antiques, et demeure connu aujourd'hui comme l'érudit qui déchiffra l'inscription sur la Maison Carrée à Nîmes en étudiant les trous laissés par les clous ${ }^{76}$. Mais c'était aussi un botaniste éminent, auteur en 1746 d'une Bibliotheca botanica, qui construisit un beau jardin des plantes autour de sa maison de ville en $1768^{77}$. Ainsi, Calvet et Séguier parlaient principalement d'antiquités, Amoreux et Séguier principalement de botanique et d'agronomie. De temps en temps, leurs conversations épistolaires pouvaient envahir un territoire étranger: comme nous l'avons vu ci-dessus, Séguier pouvait demander à Calvet de lui chercher des semences. Mais en général, les frontières restaient imperméables.

Ce que cette brève comparaison suggère n'est pas que le monde républicain de Calvet fût identique à celui des autres médecins érudits de moindre importance du Midi. Il existe bien sûr d'autres citoyens-médecins qui ne partagent ni ses intérêts intellectuels ni son engagement dans son métier. De fait, le cas d'Amoreux, sinon celui de Bouchet, révèle que Calvet n'est pas typique, même dans sa répugnance à publier. Amoreux, toujours soucieux de présenter ses idées au public, écrit constamment sur une pléthore de sujets, même si ce qu'il publie n'était pas toujours d'un grand mérite ${ }^{78}$. Ce qui unit les citoyens-médecins de talent modeste sont les limitations géographiques de leur monde. Ils jouent un rôle actif dans la République des Lettres - ils comptent parmi ses 30000 citoyens -, mais sur une petite scène provinciale. Leurs noms sont souvent connus des grands citoyens et, de temps en temps, leurs idées trouvent une plate-forme dans les grands périodiques internationaux. Toutefois, ils sont pour la plupart consommateurs des activités intellectuelles des patrons de la République. Plutôt que par leurs travaux créatifs, c'est par leurs bibliothèques qu'ils marquent leur adhésion à la République ${ }^{79}$.

75 BMA Ms 2364, lettres de Séguier à Calvet, 1760-1784; Bibliothèque Municipale Nîmes, Ms 140, lettres de Calvet à Séguier, 1760-1784; BN Ms fr. n.a. 6571, fos 112-240: lettres de Séguier à Amoreux, 1772-1784.

76 Séguier 1759.

77 Desallier d'Argenville 1780, I, 287-290: description de sa collection d'histoire naturelle et son jardin.

78 Dans les seules années 1780, Amoreux publia une bibliographie vétérinaire, des mémoires sur les oliviers, les lichens, et les haies et un grand livre sur les insectes venimeux (1789). En même temps, il produisit quatre mémoires pour la Société Royale de Médecine: voir ci-dessus la note 73 .

79 Calvet laissa une bibliothèque de 1536 titres: voir Brockliss 2002, 281-300. Il s'agit d'une grande bibliothèque pour un médecin provincial, quoiqu'elle fût petite en comparaison de celle de Haller (Brockliss 2002, 294sq.). 
De plus, s'ils contribuent à l'avancement de la science, c'est normalement en aidant les patrons de la République à développer leurs propres recherches grâce à l'accumulation de renseignements et de matériaux. A cet égard, ils sont les travailleurs à domicile de la République des Lettres par excellence. Vicq d'Azyr cultive ses relations avec Calvet et Amoreux pour construire sa carte épidémiologique de la France; Caylus utilise Calvet pour trouver des objets celto-romains afin d'illustrer son Recueil. Et les érudits provinciaux fournissent ces services, non pas par compulsion, mais pour obtenir une reconnaissance publique aux yeux du monde intellectuel parisien, et par conséquent faire élever leur statut dans leur propre sphère régionale ${ }^{80}$.

Autrement dit, cette étude de la vie intellectuelle d'un citoyen-médecin modeste démontre que notre point de départ n'est pas suffisant. En plus de faire une distinction entre les citoyens actifs et passifs, il convient de diviser la République active elle-même en deux parties et distinguer entre un petit groupe de savants internationaux, qui demeurent pour la plupart dans les cités capitales, et la grande majorité des citoyens provinciaux. Ces derniers peuplent une multiplicité de petits cercles régionaux et privés, dont les membres se choisissent en fonction de leurs intérêts intellectuels communs, et restent indépendants et autonomes, même s'ils ne sont jamais isolés, du fait que leurs membres peuvent appartenir à plus d'un seul club. En théorie, la République des Lettres constitue un état unitaire, mais en réalité, dans la deuxième moitié du XVIII ${ }^{\mathrm{e}}$ siècle, elle est faite d'une myriade de petits états entrelacés ne reconnaissant aucune autorité externe. Les cent académies peuvent inviter des républicains à répondre aux questions scientifiques qu'elles posent; elles peuvent couronner, voire faire publier la meilleure réponse; elles peuvent honorer un étranger du titre de membre correspondant. Mais elles ne peuvent ni organiser ni menacer les petites républiques autonomes dont les membres sont liés par le commerce épistolaire mutuel.

\section{Bibliographie}

Alembert, Jean Le Rond d', «Réflexions sur l'état présent de la République des lettres pour l'article gens de lettres», dans: Jean Le Rond d'Alembert, Euvres et correspondance, éd. Charles Henry (Paris 1887) 67-80

Barras, Vincent/Martin Dinges (éds), Maladies en lettres (Genève 2005, à paraître)

Blanning, Tim, The Culture of Power and the Power of Culture. Old Regime Europe, 1660-1789 (Oxford 2002)

Boschung, Urs/Barbara Braun Bucher/Stefan Hächler/Anne Kathrin Ott/Hubert Steinke/ Martin Stuber (Hrsg.), Repertorium zu Albrecht von Hallers Korrespondenz 1724-1777, 2 Bde. (Basel 2002)

80 En vieillissant, Calvet rejeta ce rôle d'agent de terrain. Après la mort de Caylus en 1765, il ne chercha plus à trouver un autre patron parisien. 
Bots, Hans/Françoise Waquet, La République des Lettres (Paris 1977)

Bouvet, Maurice, «Un remède secret du XVIII siècle: Le Rob Boyveau-Laffecteur», Bulletin de la Société d'histoire de la pharmacie 4 (1923)

Brockliss, Laurence, "Consultation by Letter in Early Eighteenth-Century Paris: The Medical Practice of Etienne-François Geoffroy", in: Ann La Berge/Mordechai Feingold (eds), French Medical Culture in the Nineteenth Century (Amsterdam 1994) 79-119

- Calvet's Web. Enlightenment and the Republic of Letters in Eighteenth-Century France (Oxford 2002)

- Colin Jones, The Medical World of Early Modern France (Oxford 1997)

Broman, Thomas, "The Habermasian Public Sphere and Science in the Enlightenment", History of Science 36 (1998) 123-149

Calvet, Esprit, Dissertation sur un monument singulier des utriculaires de Cavaillon, où l'on éclaircit un point important de la navigation des anciens (Avignon 1766)

Desallier d'Argenville, Antoine-Nicolas, La Conchyliologie, ou histoire naturelle des coquilles de mer, d'eau douce, terrestres et fossiles, 3 vols (Paris ${ }^{3} 1780$ )

Duden, Barbara, Geschichte unter der Haut. Ein Eisenacher Arzt und seine Patientinnen um 1730 (Stuttgart 1987)

Goldgar, Ann, Impolite Learning: Conduct and Community in the Republic of Letters, 1680-1750 (London 1995)

Goodman, Dena, The Republic of Letters. A Cultural History of the French Enlightenment (London 1994)

Habermas, Jürgen, The Structural Transformation of the Public Sphere: An Enquiry into the Category of Bourgeois Society, traduction anglaise (Cambridge, Mass. 1989)

Hannaway, Caroline, Medicine, Public Welfare and the State: The Société Royale de Médecine de Paris (1776-1793) (Ph.D. dissertation, Johns Hopkins University 1974)

Kafker, Frank A., The Encyclopedists as a Group. A Collective Biography of the Authors of the Encyclopédie (Oxford 1996)

McClellan III, James E., Science Reorganized. Scientific Societies in the Eighteenth Century (New York 1985)

Miller, Peter N., Peiresc's Europe: Learning and Virtue in the Seventeenth Century (London 2000)

Mosele, Elio, Un accademico francese dell settecento e la sua biblioteca (Jean-François Séguier, 1703-1784) (Verona 1981)

Moulinas, René, L'Imprimerie, la librairie et la presse à Avignon au XVIII siècle (Grenoble 1974)

Roche, Daniel, Le Siècle des lumières. Académies et académiciens provinciaux, 1680-1789, 2 vols (Paris 1978)

- "Correspondance et voyage au XVIII siècle: Le réseau des sociabilités d'un académicien provincial», dans: Daniel Roche, Les Républicains des lettres. Gens de culture et lumières au XVIII siècle (Paris 1987) chap. 11

Schnalke, Thomas, Medizin im Brief. Der städtische Arzt des 18. Jahrhunderts im Spiegel seiner Korrespondenz (Stuttgart 1997)

Séguier, Jean-François, Dissertation sur l'ancienne inscription de la Maison Carrée de Nismes (Paris 1759)

Jean-François Séguier (1703-1784). Un savant nimois dans l'Europe des Lumières, Actes du colloque international tenu à Nîmes les 17 et 18 octobre 2003 (à paraître)

Stolberg, Michael, Homo patiens. Krankheits- und Körpererfahrung in der Frühen Neuzeit (Köln, etc. 2003)

Ultee, Maarten, “The Republic of Letters: Learned Correspondence, 1680-1720”, Seventeenth Century 2 (1987) 95-112

Van Horn Melton, James, Politics, Culture and the Public Sphere in Enlightenment Europe (Cambridge 2000)

Vaughan, Gerard, "James Hugh Smith Barry as a Collector of Antiquities", Apollo (July 1987) $4-11$ 